\title{
Avaliação da Eficiência do Atendimento no Sus por meio da Análise Envoltória de Dados: um Estudo nas Microrregiões do Estado do Paraná
}

\author{
Efficiency Assessment of the Service in "sus" Through Data Envelopment Analysis: A Study in the Micro-Regions of the Paraná state
}

Evaluación de la Eficiencia de la Atención en el sus por Medio del Análisis Envoltoría de Datos: un Estudio en las Microrregiones del Estado del Paraná

Mestrado em Ciências Contábeis (Concentração em Controladoria) pela Universidade Regional de Blumenau - FURB Professor do curso de Ciências Contábeis na Universidade Comunitária de Chapecó - UNOCHAPECÓ. http://lattes.cnpq.br/1529894221455483 herivelton_schuster@hotmail.com

Suzana Habitzreuter Muller Mestrado em Ciências Contábeis (Concentração em Controladoria) pela Universidade Regional de Blumenau - FURB. Coordenadora e Professora do Curso Superior de Tecnologia em Processos Gerenciais na Faculdade SENAC - BRUSQUE SC http://lattes.cnpq.br/6024041836427892 suzanahm2013@gmail.com

Moacir Manoel Rodrigues Junior Doutorado em Métodos Numéricos em Engenharia pela Universidade Federal do Paraná - UFPR Professor do Departamento de Administração e Ciências Contábeis - FURB http://lattes.cnpq.br/7547505288125179 moacir_ro@hotmail.com

Resumo: O estudo objetivou avaliar, por meio da Análise Envoltória de Dados (DEA), a eficiência dos hospitais que atendem pelo Sistema Único de Saúde (SUS) nas microrregiões do estado do Paraná. Para tal, realizou-se uma pesquisa descritiva com abordagem quantitativa por meio da pesquisa documental. A população e amostra foram compostas por 39 microrregiões de saúde contemplando todos os municípios do estado do Paraná. Os resultados evidenciaram que, dentre as microrregiões analisadas, não se identificou discrepância significativa quanto aos scores de eficiência, ou seja, que todas se apresentaram eficientes ou próximas à fronteira de eficiência, podendo-se inferir que os recursos físicos disponibilizados ao atendimento do SUS têm sido utilizados de forma satisfatória, contribuindo assim com a qualidade de vida da população do estado do Paraná. Conclui-se que os repasses de recursos do SUS reduzem a ineficiência, reflexos dos avanços alcançados pela gestão do SUS.

Palavras-chave: Administração Hospitalar, Análise Envoltória de Dados, Sistema Único de Saúde, Saúde Pública.

\begin{abstract}
The study aimed to evaluate, using data envelopment analysis (DEA), the efficiency of hospitals that attend the brazilian national health system (Sistema Único de Saúde - SUS) in the microregions of the state of Paraná. For that, a descriptive research with a quantitative approach was done through documentary research. The population and sample were composed of 39 health microregions, contemplating all the cities of Paraná state. The results showed that among the microregions analyzed, there was no significan discrepancy regarding efficiency scores. In othe words, all of them were efficient or closely to the efficiency frontier, and it is possible to afirm that the physical resources available on SUS have been used in a satisfactory way,contributing to the life quality of the Paraná State population. In conclusion, the onlendings of SUS resources reduce inefficiency, which is a reflect of the progress reached by SUS management.
\end{abstract}

Key-words: Hospital administration, Data Envelopment Analysis, National Health System, Public Health.
Resumen: El estudio objetivó evaluar, por medio del Análisis Envoltorio de Datos (DEA), la eficiencia de los hospitales que atienden por el Sistema Único de Salud (SUS) en las microrregiones del estado de Paraná. Para ello, se realizó una investigación descriptiva con abordaje cuantitativo por medio de investigación documental. La población y muestra fueron compuestas por 39 microrregiones de salud contemplando todos los municipios del estado de Paraná. Los resultados evidenciaron que entre las microrregiones analizadas no se identificó discrepancia significativa en cuanto a los scores de eficiencia, es decir, que todas se presentaron eficientes o próximas a la frontera de eficiencia, pudiéndose inferir que los recursos físicos disponibles para la atención del SUS, se utilizaron de forma satisfactoria, contribuyendo así a la calidad de vida de la población del estado de Paraná. Se concluye que los traspasos de recursos del SUS reducen la ineficiencia, reflejos de los avances logrados por la gestión del SUS.

Palabras clave: Administración Hospitalaria Análisis de la información; Sistema único de Salud; Salud pública.

Texto completo em português: http://www.apgs.ufv.br Full text in Portuguese: http://www.apgs.ufv.br

\section{INTRODUÇÃO}

É perceptível o gradativo aumento na intenção de se analisar a eficiência hospitalar e dos serviços prestados pelo setor público, pois envolvem recursos estritamente escassos, de difícil acesso, além de ser um setor envolto de complexidades (Jacobs, 2001). Neste sentido, a eficiência do atendimento e prestação de serviços à saúde tem sido alvo de críticas em um contexto geral (Fonseca \& Ferreira, 2009).

A avaliação da eficiência dos serviços prestados à saúde, por estes serem relevantes, quando envolve recursos públicos para seu financiamento, torna-se ainda mais pertinente, haja vista que o setor de saúde é um dos principais receptores de recursos da administração pública brasileira (Marinho, 2003). Conforme Nunes e Harfouche (2015), a medida de eficiência é indispensável para a avaliação de desempenho hospitalar, pois é um critério econômico e está atrelado à maximização dos objetivos produtivos e do lucro, além dos valores sociais.

Sanderson (1996) infere que, em meio a tantas discussões, destacam-se os aspectos voltados às formas de se alcançar resultados satisfatórios para os serviços que envolvem recursos públicos. Contudo, é necessário frisar que a escassez dos recursos financeiros destinados à saúde reflete substancialmente na qualidade dos serviços prestados. Destaca-se, ainda, a dificuldade no gerenciamento por parte dos hospitais, visto que estes possuem

Correspondência/Correspondence: Herivélton Antônio Schuster. Universidade da Região de Chapecó - UNOCHAPECÓ. Departamento de Ciências Sociais Aplicadas - ACSA Servidão Anjo da Guarda, ํㅡ 295-D, Bairro Efapi - CEP: 89809-900. Chapecó SC herivelton_schuster@hotmail.com 
estrutura organizacional complexa e necessitam treinar e profissionalizar os colaboradores para a prestação dos serviços (Hames, 1991).

Ainda no que tange o gerenciamento, a presença de fatores tanto externos quanto internos é responsável pela complexidade do ambiente hospitalar. Como exemplo tem-se a contenção de recursos dos agentes financiadores do sistema e a busca pela excelência e qualidade no atendimento aos cidadãos. Uma forma de amenizar tais interferências e impactos é ser eficiente no processo de atendimento e gestão de recursos (Hames, 1991).

Behn (1998) destaca características atreladas à administração pública e que refletem na eficiência exigida pela população, como a lentidão e a burocracia. Outras características deste setor são os custos operacionais elevados e fontes de receitas incertas e geralmente insuficientes (Marinho, 2003; Mills \& Spancer, 2005).

A limitação dos recursos destinados à saúde reflete no interesse dos gestores das redes públicas e privadas, bem como da sociedade que está exposta aos serviços prestados, em avaliar a eficiência e o desempenho dos hospitais. Isso é decorrente das incertezas inerentes aos recursos disponibilizados, sendo viável analisar se estão sendo devidamente utilizados e aplicados nas redes hospitalares (Wolff, 2005).

Smith e Streert (2005) destacam a dificuldade e desafio que envolve a mensuração da eficiência do atendimento dos serviços de saúde. Os autores inferem que se trata de um trabalho pertinente, tendo em vista o ambiente com elevado nível de complexidade e influenciado por diferentes fatores. Contudo, para tal mensuração deve-se atentar aos fenômenos observáveis, bem como à percepção da relação entre eles, o índice de eficiência máxima e a avaliação dos resultados, assim como seus reflexos na ineficiência (Smith \& Streert, 2005).

Neste sentido, tem-se a pergunta que norteia o desenvolvimento desta pesquisa: Qual o nível de eficiência dos hospitais que atendem pelo Sistema Único de Saúde (SUS) nas microrregiões do estado do Paraná? Com isso, o estudo objetiva avaliar, por meio da Análise Envoltória de Dados (DEA), a eficiência dos hospitais que atendem pelo SUS das microrregiões do estado do Paraná.

O estudo justifica-se principalmente pela relevância e pertinência do assunto para a sociedade como um todo. Ampliar os estudos já existentes na literatura, como o de Cesconetto, Lapa e Calvo (2008), que analisaram a eficiência de 112 hospitais do estado de Santa Catarina, abordando aspectos de recursos humanos, recursos materiais, recursos financeiros de produtos hospitalares, trazendo uma ligação importante entre academia e gestão pública de forma geral. Outros estudos importantes são de Souza, Nishijima e Rocha (2010), o qual avaliaram a eficiência produtiva de hospitais do estado de São Paulo; Politelo e Scarpim (2013), que avaliaram a questão da eficiência do atendimento do SUS das vinte microrregiões do estado de Santa Catarina, por meio da análise envoltória de dados (DEA); Politelo, Rigo e Hein (2015), os quais analisaram a eficiência da aplicação de recursos no atendimento do Sistema Único de Saúde (SUS) nos municípios do estado de Santa Catarina; Kaveski, David, Degenhart, Vogt e Hein (2015), que buscaram identificar o nível de eficiência do atendimento do SUS no Sudoeste de Mato Grosso do Sul. E também o de Santos, Souza Francisco e Gonçalves (2016), os quais objetivaram investigar a eficiência da alocação de recursos públicos na atenção primária da saúde, tomando como referência as microrregiões do Sudeste do Brasil. No entanto, este estudo diferencia-se por analisar a eficiência do atendimento do SUS por meio de um conjunto diferenciado de variáveis e num contexto diferente dos já analisados.

O estudo visa gerar resultados que poderão contribuir aos municípios menos eficientes, pois será possível indicar padrões de eficiência a serem espelhados por meio dos benchmarks e no que tange os recursos públicos, bem como orientará quanto às políticas emprego dos recursos disponíveis no atendimento público de saúde. Justifica-se, ainda, pela busca constante em se mensurar a eficiência das redes hospitalares com atendimentos SUS, pois segundo Marinho (2001), é financiada pelos recursos públicos e constantemente executa atendimentos de forma precária à população.

\section{REFERENCIAL TEÓRICO}

Este tópico apresenta a revisão da literatura que dá suporte ao presente estudo. Inicialmente, são abordados conteúdos referentes à gestão e qualidade dos serviços públicos de saúde. Em seguida sobre os desafios na mensuração da eficiência no setor hospitalar. E estudos correlatos ao objetivo dessa pesquisa.

\section{Gestão e qualidade dos serviços públicos de saúde}

As organizações do setor de saúde, mais especificamente os hospitais, são envoltas de alta complexidade, visto que seu objetivo principal é dar acesso à saúde para a população em geral. Pode-se ressaltar a esta atividade situações, como diagnósticos de doenças, tratamento de pacientes, reabilitação e prevenção de agravos à saúde. Cabe ainda destacar o desenvolvimento de atividades de ensino e pesquisa (Katz \& Kahn, 1975; Shaw, 2003).

Segundo Fonseca e Ferreira (2009), no contexto brasileiro, os serviços de saúde são apresentadas de forma não satisfatória às necessidades dos cidadãos, causadas possivelmente pelas influências políticas de prestação destes serviços. Tais especificidades indicam dualidade de aspetos quanto às políticas de saúde, como a privatização dos serviços de saúde, resultando na dificuldade de acesso pela maior parte da população à saúde e a necessidade óbvia da busca pela manutenção do direito conquistado na Constituição Federal, de 1988, a qual garante acesso igualitário a serviços de qualidade (Fonseca \& Ferreira, 2009)

Contudo, Morrisey, Wedig e Hassan (1996) inferem que as redes hospitalares e que prestam serviços à população são envoltas de limitações, principalmente quando se trata de recursos disponíveis, sejam eles monetários ou físicos, os quais afetam claramente o seu desempenho e as expectativas da população. Apesar de tais limitações de recurso, algumas organizações tendem a ignorar as limitações dos recursos, visando o bom atendimento, visto que seu objetivo é a preservação da vida. No entanto, para que isso aconteça, não pode ser descartada a existência de recursos, uma 
vez que estes são essenciais para a manutenção dos fluxos de caixa da entidade e longevidade de toda empresa (Morrisey et al., 1996).

Neste sentido, faz-se necessário ressaltar que o conceito de qualidade atrelado ao atendimento no setor da saúde pode variar entre culturas ou grupos de indivíduos (Fadel \& Regis Filho, 2009). Segundo Castelar, Mordelet e Grabois (2003), o alcance de uma gestão eficiente e a prestação de serviços de qualidade está atrelada à utilização de combinação ótima dos recursos humanos, tecnológicos e financeiros.

No que tange à qualidade dos serviços de saúde prestados, temse que está totalmente interligada à escassez de recursos, físicos ou financeiros (Marinho, 2003; Mills \& Spancer, 2005). De modo geral, quando se tratam de serviços públicos, geralmente são caracterizados por serem ineficientes e não produtivos, ou seja, a sociedade enxerga-os de forma pessimista, e, dessa forma, a reestruturação na gestão pública torna-se primordial para satisfazer as necessidades deste cenário de incerteza e desconfiança (Mwita, 2000).

Tal realidade é presenciada por organizações de diversos setores, não podendo condicionar apenas ao da saúde, no entanto está atrelada a este setor em detrimento de tantas especificidades no que tange os serviços prestados (Malik \& Teles, 2001). Com isso, o setor hospitalar é percebido com desiquilíbrio e conflitos, haja vista que está submisso a custos elevados para os tratamentos e em contrapartida as receitas geralmente insuficientes (Malik \& Teles, 2001; Mills \& Spancer, 2005).

\section{Desafios na mensuração da eficiência e métodos}

A avaliação de desempenho nos hospitais está ligada à maximização dos lucros nos hospitais privados, dos valores sociais e dos objetivos do processo produtivo, sendo que a eficiência, economicamente caracterizada, concebe uma dimensão imprescindível para tal avaliação (Simon, 1957).

Neste sentido, Marinho (2003) destaca que a avaliação da eficiência nos serviços prestados pelas organizações públicas é muito importante, com destaque à área da saúde pública, pois é identificada como um dos principais destinos dos recursos financeiros repassados pela administração pública. Para tanto, a mensuração da eficiência se dá pela diferença entre os resultados recebidos em relação aos resultados aplicados, visando, por parte dos hospitais, a prestação de serviços com vasta apreciação pela sociedade, tendo como base o menor custo na prestação do serviço (Cebrián, 1995).

No mesmo sentido, Chuang, Chang e Lin (2011) definem a mensuração da eficiência como sendo o nível mínimo de utilização das entradas frente a certas quantias de saídas. Os autores Viacava et al. (2012) destacam que, na área da saúde, a eficiência é manter o nível de qualidade gerenciando a relação do custo versus seu reflexo na saúde populacional.

Visando o alcance da eficiência dos serviços prestados pelos hospitais, tem-se que os relatórios de desempenho são utilizados como ferramentas imprescindíveis para sua mensuração, além de serem utilizados para controle e monitoramento dos serviços prestados (Greiling, 2006).
Cabe destacar que há fatores que dificultam a mensuração da eficiência hospitalar. Moreira (2008) destaca que a multiplicidade de objetivos e as definições dos processos produtivos podem impactar o estado de saúde da população. No entanto, a complexidade existente nos hospitais e o grande volume financeiro aplicado têm influenciado para o incremento e utilização de mecanismos que maximizem a qualidade dos serviços prestados, sem perder o foco da eficiência no emprego dos recursos (Souza \& Scatena, 2010).

Uma forma de aumentar a eficiência e eficácia nos serviços prestados, a partir de recursos públicos, segundo Andrews e Entwistle (2010), é a parceria público-privada, considerada uma prática corriqueira no setor hospitalar. Essas parcerias visam suprir a escassez de recursos e desenvolver uma melhora na qualidade da gestão.

Smith e Street (2005) discorrem sobre a necessidade de compreender os limites estabelecidos pela análise da eficiência dos serviços públicos. Além disso, existe uma demanda na avaliação e medição da eficiência dos serviços atrelados à utilização de recursos públicos a partir dos gestores das organizações. Para tanto, o método de análise envoltória de dados (DEA) é considerada uma metodologia capaz de responder de forma adequada esta necessidade.

\section{Estudos Correlatos}

Al-Shammari (1999) avaliou e mensurou a eficiência de hospitais situados na Jordânia no período de 1991 a 1993, por meio da Análise Envoltória de Dados (DEA). Os resultados demonstraram que grande parte dos hospitais analisados são eficientes e que aqueles que não são eficientes estão próximos da fronteira de eficiência. $\mathrm{O}$ autor conclui também que os hospitais analisados são eficientes quanto à utilização dos recursos e que a eficiência poderia ser melhorada a partir da divulgação à sociedade sobre os hospitais que não estão utilizando sua capacidade total.

No estudo de Kirigia et al. (2004), foi analisada a eficiência utilizando-se uma base de dados de 32 centros públicos de saúde do Quênia, e por meio do método DEA. Os resultados demonstram ineficiência de $44 \%$ da amostra pesquisada. Também identificaram alternativas para melhoria da eficiência na utilização dos recursos públicos, como a redução do pessoal ligado à área administrativa, a transferência de equipamentos dos centros ineficientes para aqueles considerados eficientes, bem como sua venda ou locação para empresas privadas.

Cesconetto et al. (2008) objetivaram verificar a eficiência em 112 hospitais conveniados ao SUS no ano de 2003 no estado de Santa Catarina. Utilizando-se do método DEA chegaram à conclusão que aproximadamente $21 \%$ dos hospitais são eficientes e que a eficiência poderia ser melhorada a partir do aumento dos atendimentos, bem como com a redução de em $25 \%$ do pessoal ligado à enfermagem. Também indicaram a redução de 17\% da quantidade de leitos disponíveis e redução de $13 \%$ do valor das Autorizações de Internação Hospitalar (AlH) como meio de obterem eficiência.

Em seus estudos, Clement et al. (2008) verificaram a eficiência de 10 hospitais nos estados norte-americanos, no ano 2000, por 
meio do modelo DEA. Os resultados evidenciaram que a ineficiência está associada à má qualidade no atendimento dos pacientes, sendo este motivo, na maior parte dos hospitais uma possibilidade para melhorar a eficiência. Inferiram também que 33\% dos hospitais analisados adaptaram-se às limitações de recursos sem prejudicar a qualidade do atendimento.

Souza et al. (2010) analisaram hospitais em 366 municípios do estado de São Paulo. O estudo objetivou avaliar o grau de eficiência produtiva do setor de público no período de 1998 a 2003. Os resultados demonstraram que os municípios menos eficientes são os com maior população, também que são mais eficientes os que contratam um maior número de leitos de hospitais privados e de leitos universitários, que possuem menor população, maior número de internações e menor tempo médio de internações.

O estudo de Politelo e Scarpin (2013) objetivou analisar a eficiência do atendimento do SUS das vinte microrregiões do estado de Santa Catarina através da Análise Envoltória de Dados (DEA). Os resultados demonstram que $65 \%$ das microrregiões pertencentes à amostra são eficientes. Contudo, a média de eficiência do estado de Santa Catarina é de $97,04 \%$, sendo que $75 \%$ das microrregiões apresentam eficiência superior à média do estado, e a microrregião menos eficiente apresentou escore de eficiência igual a 0,786 , ou $78,6 \%$. Conclui-se que o SUS é eficiente quanto ao atendimento no estado de Santa Catarina.

Politelo et al. (2015) avaliaram a eficiência na alocação de recursos no do Sistema Único de Saúde (SUS) pelos municípios do estado de Santa Catarina. Para tanto, utilizaram o método de Análise Envoltória de Dados (DEA). Os resultados demonstraram que $28 \%$ dos municípios analisados desempenharam eficiência no que tange a utilização dos recursos disponíveis ao SUS e que a eficiência média do estado de SC é de $86,26 \%$. Detectaram ainda a eficiência da aplicação de recursos no atendimento do SUS de Santa Catarina em comparação aos achados de estudos anteriores. Concluíram, então, que a eficiência da aplicação de recursos no atendimento do SUS nas cidades catarinenses é satisfatória, porém, pode haver uma melhora a partir da orientação das cidades não eficientes, pelos seus benchmarks explanados na análise do estudo.

Kaveski et al. (2015) buscaram identificar o nível de eficiência do atendimento do SUS no Sudoeste de Mato Grosso do Sul, por meio da análise envoltória de dados (DEA). Os resultados indicam que apenas $31 \%$ dos municípios da amostra são eficientes. Entretanto, $54 \%$ dos municípios pesquisados demonstraram uma eficiência acima da média encontrada na mesorregião do estado. Concluíram, contudo, que a eficiência da utilização dos recursos destinados às unidades de saúde vinculadas ao SUS pertencentes ao Sudoeste do Mato Grosso do Sul é satisfatória.

Santos et al. (2016) tiveram por objetivo investigar a eficiência da alocação de recursos públicos na atenção primária da saúde, tomando como referência as microrregiões do Sudeste do Brasil. Os resultados demonstraram um mediano desempenho das microrregiões do Sudeste, e o Estado de São Paulo apresentou o maior número de unidades eficientes. Denota-se a importância de utilização de ferramentas de controle baseando-se em resultados instigando a criação de novas políticas para a melhoria da eficiência do atendimento primário em saúde, que consideram as diversidades dos municípios e microrregiões do país, em função das particularidades inter-regionais.

A Tabela 1 expõe as variáveis de inputs e outputs utilizados para cada estudo supracitado.

Tabela 1: Variáveis utilizadas pelos estudos correlatos

\begin{tabular}{|c|c|c|c|}
\hline Autor & Método & Inputs & Outputs \\
\hline $\begin{array}{l}\text { Al-Shammari } \\
(1999)\end{array}$ & DEA & Dias de internação. Número de médicos. Profissionais da saúde. & Pacientes diários. Pequenas cirurgias. Grandes cirurgias. \\
\hline $\begin{array}{l}\text { Kirigia et al. } \\
\quad(2004)\end{array}$ & DEA & $\begin{array}{l}\text { Médicos e enfermeiros. Fisioterapeuta, terapeuta ocupacional, agente } \\
\text { de saúde e dentista. Técnico de laboratório. Administrativo. Despesas } \\
\text { operacionais. Número de leitos. }\end{array}$ & $\begin{array}{l}\text { Procedimentos não cirúrgicos (tratamentos e prevenções, } \\
\text { infecções e doenças respiratórias). Pré-natal e assistência } \\
\text { familiar. Imunização. Outros procedimentos ambulatoriais. }\end{array}$ \\
\hline $\begin{array}{l}\text { Cesconetto et } \\
\text { al. (2008) }\end{array}$ & DEA & $\begin{array}{l}\text { Médicos e profissionais auxiliar de enfermagem. Número de leitos } \\
\text { SUS. Valor das AlH. }\end{array}$ & $\begin{array}{l}\text { Número de altas geradas pelo hospital para pacientes do } \\
\text { SUS. }\end{array}$ \\
\hline $\begin{array}{l}\text { Clement et al. } \\
\quad(2008)\end{array}$ & DEA & $\begin{array}{l}\text { Número de enfermeiro. Número de auxiliar de enfermagem. Outros } \\
\text { Profissionais. Leitos em Utilização. }\end{array}$ & $\begin{array}{l}\text { Número de partos. Cirurgias ambulatoriais. Atendimentos } \\
\text { de emergência. Consultas externas. Outros procedimentos } \\
\text { cirúrgicos. }\end{array}$ \\
\hline $\begin{array}{l}\text { Souza et al. } \\
\quad(2010)\end{array}$ & FDF & $\begin{array}{l}\text { Pessoal. Leitos. Tendência de internações. Internações per Capita. } \\
\text { Gestão política - PMDB; PSDB; PT; PFL; PTB e demais Partidos. } \\
\text { Número de internações em: hospitais públicos, hospitais privados, } \\
\text { hospitais universitários, clínica cirúrgica, obstetrícia, clínica médica, } \\
\text { pediatria, outras internações. Gasto público. }\end{array}$ & $\begin{array}{l}\text { Mortalidade hospitalar. } \\
\text { Taxa de permanência. }\end{array}$ \\
\hline $\begin{array}{c}\text { Politelo e } \\
\text { Scarpin (2013) }\end{array}$ & DEA & $\begin{array}{l}\text { Capacidade ambulatorial. Valor médio das internações. Quantidade } \\
\text { de leitos por hospitais. }\end{array}$ & $\begin{array}{l}\text { Internações per capita. Total de procedimentos } \\
\text { ambulatoriais per capita. Inverso da taxa de mortalidade. }\end{array}$ \\
\hline $\begin{array}{l}\text { Politelo et al. } \\
\text { (2015) }\end{array}$ & $\overline{D E A}$ & $\begin{array}{l}\text { Capacidade ambulatorial (Equipamentos e Pessoal). Valor médio das } \\
\text { internações. Leitos por hospital. }\end{array}$ & $\begin{array}{l}\text { Internações per capita. Total de procedimentos } \\
\text { ambulatoriais per capita. Inverso da taxa de mortalidade. }\end{array}$ \\
\hline $\begin{array}{l}\text { Kaveski et al. } \\
\text { (2015) }\end{array}$ & DEA & $\begin{array}{l}\text { Capacidade ambulatorial. Valor médio das internações. Número de } \\
\text { Leitos per capita. }\end{array}$ & $\begin{array}{l}\text { Internações per capita. Produção Ambulatorial per capita. } \\
\text { Inverso da Taxa de Mortalidade }\end{array}$ \\
\hline $\begin{array}{l}\text { Santos et al. } \\
\quad(2016)\end{array}$ & DEA & $\begin{array}{l}\text { Cobertura da atenção básica. Cobertura de acompanhamento das } \\
\text { condicionalidades de saúde do Programa Bolsa Família. Cobertura } \\
\text { populacional estimada pelas equipes básicas de saúde bucal. Número } \\
\text { de Equipes de Saúde per capita. }\end{array}$ & $\begin{array}{l}\text { Inverso da proporção de internações por condições } \\
\text { sensíveis à atenção primária (ICSAB). Inverso da taxa de } \\
\text { mortalidade infantil. }\end{array}$ \\
\hline
\end{tabular}

Fonte: Dados da pesquisa (2015).

Legenda: Análise Envoltória dos Dados - DEA; Flexível de Fourier - FDF; Sistema Único de Saúde - SUS; Tempo de uso dos leitos - FTS;

\section{PROCEDIMENTOS METODOLÓGICOS}

O presente estudo tem como objetivo avaliar, por meio da Análise Envoltória de Dados (DEA), a eficiência dos hospitais que atendem pelo Sistema Único de Saúde (SUS) das microrregiões do estado do Paraná. Neste contexto, caracteriza-se com tipologia descritiva, visando descrever a eficiência da amostra analisada
(Vergara, 2000). Quanto à abordagem do problema, quantitativa, pois conforme Richardson (1999) se utiliza de tratamento estatístico para análise dos dados. Também se caracteriza como documental por embasar-se em informações públicas como relatórios divulgados pela secretaria da saúde. 
A população da pesquisa constituiu-se de 416 hospitais pertencentes às 39 microrregiões de saúde, contemplando todos os municípios do Paraná. Destes, foram obtidos os dados de todos os hospitais vinculados ao Sistema Único de Saúde (SUS). A amostra da pesquisa foi composta, levando em consideração as instituições

Tabela 2:Total da população e amostra do estudo

\begin{tabular}{|c|c|c|c|}
\hline Microrregiões & $\begin{array}{l}\text { Total de Municípios } \\
\text { da Microrregião }\end{array}$ & $\begin{array}{c}\text { Total de } \\
\text { Municípios da } \\
\text { Amostra }\end{array}$ & $\begin{array}{c}\text { Total de } \\
\text { Hospitais da } \\
\text { Amostra }\end{array}$ \\
\hline Apucarana & 9 & 3 & 6 \\
\hline Assaí & 8 & 4 & 4 \\
\hline Astorga & 22 & 10 & 10 \\
\hline Campo Mourão & 14 & 6 & 8 \\
\hline Capanema & 8 & 6 & 7 \\
\hline Cascavel & 18 & 10 & 14 \\
\hline Cerro Azul & 3 & 1 & 1 \\
\hline Cianorte & 11 & 4 & 5 \\
\hline $\begin{array}{l}\text { Cornélio } \\
\text { Procópio }\end{array}$ & 14 & 9 & 10 \\
\hline Curitiba & 19 & 13 & 27 \\
\hline Faxinal & 7 & 2 & 2 \\
\hline Florai & 7 & 6 & 6 \\
\hline Foz do Iguaçu & 11 & 6 & 8 \\
\hline $\begin{array}{l}\text { Francisco } \\
\text { Beltrão }\end{array}$ & 19 & 9 & 12 \\
\hline Goioerê & 11 & 8 & 8 \\
\hline Guarapuava & 18 & 6 & 9 \\
\hline Ibaiti & 8 & 5 & 5 \\
\hline Irati & 4 & 3 & 4 \\
\hline Ivaiporã & 15 & 8 & 10 \\
\hline Jacarezinho & 6 & 3 & 4 \\
\hline Jaguariaíva & 4 & 3 & 3 \\
\hline Lapa & 2 & 1 & 1 \\
\hline Londrina & 6 & 5 & 12 \\
\hline Maringá & 5 & 4 & 10 \\
\hline Palmas & 5 & 3 & 3 \\
\hline Paranaguá & 7 & 5 & 5 \\
\hline Paranavaí & 29 & 11 & 12 \\
\hline Pato Branco & 10 & 4 & 5 \\
\hline Pitanga & 6 & 1 & 1 \\
\hline Ponta Grossa & 4 & 3 & 9 \\
\hline Porecatu & 8 & 7 & 7 \\
\hline Prudentópolis & 7 & 4 & 6 \\
\hline Rio Negro & 6 & 4 & 4 \\
\hline $\begin{array}{l}\text { São Mateus do } \\
\text { Sul }\end{array}$ & 3 & 2 & 2 \\
\hline $\begin{array}{l}\text { Telêmaco } \\
\text { Borba }\end{array}$ & 6 & 4 & 4 \\
\hline Toledo & 21 & 11 & 15 \\
\hline Umuarama & 21 & 7 & 9 \\
\hline $\begin{array}{l}\text { União da } \\
\text { Vitória }\end{array}$ & 7 & 3 & 3 \\
\hline $\begin{array}{l}\text { Wenceslau } \\
\text { Braz }\end{array}$ & 10 & 8 & 8 \\
\hline Total & 399 & 219 & 290 \\
\hline
\end{tabular}

Fonte: Dados da pesquisa (2015).

Para uma análise fidedigna, foram identificadas as microrregiões com características que os diferenciam dos demais, visto que estas podem distorcer os resultados se tratando da análise de eficiência, aplicando-se, para tanto, a técnica estatística de Clusters. Conforme Corrar, Paulo e Dias Filho (2007) esta técnica possibilita o agrupamento dos objetos, neste caso as microrregiões de saúde, a partir de suas características semelhantes. Neste sentido, a técnica será aplicada em relação aos inputs, estratificando, assim, a amostra, e garantindo melhores comparativos dos resultados.

Os dados foram obtidos do Sistema de Processamento de Dados do Sistema Único de Saúde (DATASUS) e CNESNet, contemplando o período do ano 2014 ao primeiro semestre do ano 2015, em virtude de ser o período mais recente com dados disponíveis. A Tabela 3 relaciona os inputs e outputs utilizados no estudo. denominadas "Hospital Geral" que pertencem aos municípios de cada microrregião do estado, conforme Tabela 2. Ressalta-se que foram excluídos da amostra os municípios em que todos os hospitais não apresentavam as variáveis necessárias para análise.

Tabela 3: Variáveis utilizadas no estudo

\begin{tabular}{|c|c|c|c|}
\hline & $\begin{array}{l}\text { Nome da } \\
\text { Variável }\end{array}$ & Descrição do Inputs & Autores \\
\hline \multirow{5}{*}{ 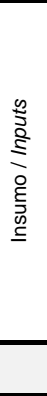 } & EQUIP & $\begin{array}{l}\text { Número de equipamentos } \\
\text { disponíveis ao SUS em } \\
\text { junho de } 2015 \text {. }\end{array}$ & Fonseca e Ferreira (2009). \\
\hline & VLRMI & $\begin{array}{l}\text { Valor médio das } \\
\text { internações. }\end{array}$ & $\begin{array}{l}\text { Kirigia et al. (2004); Politelo e } \\
\text { Scarpin (2013); Politelo et al. } \\
\text { (2015); Kaveski et al. (2015); }\end{array}$ \\
\hline & $\mathrm{AlH}$ & $\begin{array}{l}\text { Número de Autorizações } \\
\text { de Internação Hospitalar. }\end{array}$ & Souza et al. (2010); \\
\hline & NLEIT & $\begin{array}{l}\text { Número de leitos do SUS } \\
\text { em junho de } 2015 \text {. }\end{array}$ & $\begin{array}{l}\text { Kirigia et al. (2004); } \\
\text { Cesconetto et al. (2008); } \\
\text { Clement et al. (2008); Souza } \\
\text { et al. (2010); Politelo e } \\
\text { Scarpin (2013); Politelo et al. } \\
\text { (2015); Kaveski et al. (2015); }\end{array}$ \\
\hline & $\begin{array}{l}\text { Nome da } \\
\text { Variável }\end{array}$ & Descrição do Output & Autores \\
\hline \multirow{3}{*}{$\begin{array}{l}\frac{0}{2} \\
\frac{2}{5} \\
0 \\
0 \\
\frac{1}{2} \\
\frac{0}{2} \\
\frac{0}{2}\end{array}$} & PROCN & $\begin{array}{l}\text { Procedimentos não } \\
\text { cirúrgicos (ambulatoriais) } \\
\text { realizados per capita - } \\
\text { considerados as ações de } \\
\text { promoção e prevenção em } \\
\text { saúde, procedimentos com } \\
\text { finalidade, procedimentos } \\
\text { clínicos e medicamentos. }\end{array}$ & $\begin{array}{l}\text { Clement et al. (2008); Souza } \\
\text { et al. (2010); Kaveski et al. } \\
\text { (2015); }\end{array}$ \\
\hline & INTER & $\begin{array}{l}\text { Total de internações per } \\
\text { capita. }\end{array}$ & $\begin{array}{l}\text { Kirigia et al. (2004); Souza et } \\
\text { al. (2010); Politelo e Scarpin } \\
\text { (2013); Kaveski et. al. (2015); }\end{array}$ \\
\hline & IMORT & $\begin{array}{l}\text { Corresponder ao } \\
\text { complemento da variável } \\
\text { taxa de mortalidade } \\
\text { hospitalar (100 - taxa de } \\
\text { mortalidade). }\end{array}$ & $\begin{array}{l}\text { Clement et al. (2008); } \\
\text { Politelo e Scarpin (2013); } \\
\text { Politelo et al. (2015); Kaveski } \\
\text { et al. (2015); Santos et al. } \\
\text { (2016); }\end{array}$ \\
\hline
\end{tabular}
Fonte: Dados da pesquisa (2015).

Por se tratar de análise das microrregiões, alguns procedimentos foram necessários para captação das variáveis estudadas, visto que dados obtidos referem-se aos municípios de cada microrregião. Neste sentido, os procedimentos foram: i) Para a variável "EQUIP" efetuou-se a soma dos equipamentos disponíveis ao SUS de cada município; ii) Para obter a "VLRMl", calculou-se a média do valor médio das internações de cada municípios; iii) Para a variável "AlH", efetuou-se a soma das autorizações de internações aprovadas; iv) A variável "NLEIT" somou-se o número de leitos do SUS disponíveis em cada hospital para cada município, em junho de 2015; v) A variável "PROCN" foi obtida pela soma dos procedimentos não cirúrgicos dividido pelo total de habitantes da microrregião; vi) Para a "INTER", somou-se o número de internações de cada município e efetuou-se a divisão pelo número de habitantes da microrregião; vii) Por fim, para a variável "IMORT", primeiro foi subtraído de 100 a taxa de mortalidade de cada município e, posteriormente, foi calculado a média de cada microrregião, resultando, assim, na taxa inversa da mortalidade.

Após a coleta, os dados foram tabulados em planilha eletrônica. A análise teve início realizando a separação, por similaridade, dos hospitais em grupos utilizando a Análise de Clusters não hierárquico. A operacionalização deste método deu-se por meio do software SPSS. Efetuada o agrupamento, efetuou-se a mensuração da eficiência dos dados por meio da Análise Envoltória de Dados (Data Envelopment Analysis - DEA), com aplicação do modelo BCC orientado ao output (Saída), visto que o produto originado a partir dos insumos evidencia a eficiência na utilização dos recursos disponíveis a cada Unidade de Tomada de Decisões (DMU). 
Referente às análises da eficiência, foram realizadas por meio do software MaxDEA $5 \AA$.

O modelo DEA foi criado por Charnes, Cooper e Rhodes em 1978, e denominado modelo CCR. Posteriormente Banker, Charnes e Cooper em 1984, aperfeiçoaram o modelo CCR originando o modelo BCC. Com a aplicação do DEA, cada DMU determina seu próprio conjunto de pesos, com intenção de ser mais bem comparada com as demais (Mello et. al., 2005). Com isso, é possível produzir um indicador (score) que varia de 0 a 1 , em que quanto mais próximo a 1, mais eficiente é considerada a DMU, e escores iguais a 1 indicam eficiência máxima (Macedo, Barbosa \& Cavalcante, 2009).

Conforme Lins e Meza (2000), a metodologia DEA ocorre em três etapas.A primeira define as DMU's, e, neste estudo, as microrregiões de saúde. Já a segunda define as variáveis de insumo (inputs) e de produto (outputs), e a terceira ocorre com a aplicação dos modelos DEA. Os autores ressaltam as vantagens do método, que é a disponibilidade de utilização de múltiplos inputs e outputs sem que a análise torne-se complexa. Segundo Oro, Beuren e Hein (2009) essas características apresentadas acima tornam a metodologia flexível, uma vez que permite a inclusão de variáveis múltiplas de entrada e saída, e as mesmas são calculadas simultaneamente.

O modelo DEA é composto de duas partições principais: O modelo CCR, que remete a retornos constantes à escala, e resulta na comparação das DMU's entre si, independentes das especificidades de cada uma. O modelo BCC, que remete a retornos variáveis à escala, ou seja, as DMU's ineficientes, são definidas a partir do comparativo entre as da amostra, e, neste caso, as especificidades tornam-se relevantes (Hass, 2003). Neste sentido, são gerados indicadores de eficiência que variam de 0 a 1 para cada DMU, sendo que quanto mais próximo a 1 , mais eficiente esta é considerada, visto que scores iguais a 1 indicam eficiência máxima (Macedo et al., 2009).

Vale ressaltar que uma das principais funcionalidades do modelo é que ele converte várias saídas e entradas em escala de mensuração da eficiência e delineando uma fronteira paramétrica das DMUs analisadas e que, posteriormente, são utilizadas como parâmetro de eficiência quanto à comparação das mesmas (Hu, Qi, \& Yang, 2012).

Tabela 4: Eficiência das microrregiões do grupo 2

\begin{tabular}{c|c|c|l}
\hline Ordem & Microrregião & Score de Eficiência & \multicolumn{1}{c}{ Benchmark } \\
\hline 1 & Francisco Beltrão & 1 & - \\
\hline 2 & Toledo & 1 & - \\
\hline 3 & Guarapuava & 0,994 & Toledo; Francisco Beltrão; \\
\hline 4 & Foz do lguaçu & 0,993 & Toledo; \\
\hline 5 & Cascavel PR & 0,991 & Toledo; Francisco Beltrão; \\
\hline 6 & Apucarana & 0,987 & Toledo; Francisco Beltrão; \\
\hline 7 & Maringá & 0,982 & Toledo; Francisco Beltrão; \\
\hline 8 & Ponta Grossa & 0,977 & Toledo; \\
\hline 9 & Londrina & 0,968 & Toledo; Francisco Beltrão; \\
\hline
\end{tabular}

Fonte: Dados da pesquisa (2015).

Pode-se verificar, a partir da Tabela 4, que dentre as nove microrregiões que compõe o grupo 2, duas obtiveram score 1 de eficiência e sete foram menos eficientes, porém estão muito próximas à fronteira de eficiência. Vale ressaltar que para as menos

\section{ANÁLISE DOS DADOS}

A fim de cumprir com o objetivo proposto pelo estudo, como ressaltado no tópico anterior, as microrregiões da amostra foram classificadas agrupando-as por semelhança dos inputs por meio da técnica de Clusters. Para tanto, utilizou-se as variáveis EQUIP, VLRMI, AIH e NLEIT e formou-se 3 grupos: Grupo1, composto apenas pela microrregião de Curitiba; o Grupo 2, composto pelas microrregiões Apucarana, Cascavel, Foz do Iguaçu, Francisco Beltrão, Guarapuava, Londrina, Maringá, Ponta Grossa e Toledo; e - Grupo 3, composto pelas microrregiões Assaí, Astorga, Campo Mourão, Capanema, Cerro Azul, Cianorte, Cornélio Procópio, Faxinal, Floraí, Goioerê, Ibaiti, Irati, Ivaiporã, Jacarezinho, Jaguariaíva, Lapa, Palmas, Paranaguá, Paranavaí, Pato Branco, Pitanga, Porecatu, Prudentópolis, Rio Negro, São Mateus do Sul, Telêmaco Borba, Umuarama, União Vitória e Wenceslau Braz.

Neste sentido, visto que não se pode efetuar avaliação da eficiência de uma única microrregião, para o Grupo 1 (microrregião de Curitiba) foi analisado a eficiência dos 13 municípios que a compõe. Quanto às variáveis que compunham a microrregião do Grupo 1, tem-se 3.096 equipamentos disponíveis ao SUS em junho de 2015, o valor médio das internações de $\mathrm{R} \$ 2.122,57,283.313$ autorizações de internações aprovadas e realizadas, 3.711 leitos do SUS, 124.283 procedimentos não cirúrgicos (ambulatoriais) realizados, considerados as ações de promoção e prevenção em saúde, procedimentos com finalidade, procedimentos clínicos e medicamentos, e um complemento da taxa de mortalidade de 96,20.

Vale ressaltar que os números supracitados acima correspondem à soma dos inputs e outputs dos 13 municípios que compõe a microrregião do Grupo 1. Após a análise de cada DMU deste grupo, constatou-se que todas (Araucária, Bocaiúva do Sul, Campina Grande do Sul, Campo Largo, Contenda, Curitiba, Fazenda Rio Grande, Itaperuçu, Mandirituba, Pinhais, Piraquara, Rio Branco do Sul e São José dos Pinhais) apresentaram score de eficiência máximo, neste caso 1, não havendo discrepância entre os resultados de ambos.

A Tabela 4 expõe os escores de eficiência do Grupo 2 das microrregiões analisadas bem como o benchmark dos ineficientes. eficientes, pode-se fazer a comparação com os benchmarks, que neste caso se destacam as microrregiões de Toledo e Francisco Beltrão. A Tabela 5 a seguir expressa os scores de eficiência e os benchmarks dos hospitais ineficientes do grupo 3 de análise. 
Tabela 5: Eficiência das microrregiões do grupo 3

\begin{tabular}{|c|c|c|c|}
\hline Ordem & Microrregião & $\begin{array}{c}\text { Score de } \\
\text { Eficiência }\end{array}$ & Benchmark \\
\hline 1 & Assaí & 1 & - \\
\hline 2 & Campo Mourão & 1 & - \\
\hline 3 & Cerro Azul & 1 & - \\
\hline 4 & Cianorte & 1 & - \\
\hline 5 & Faxinal & 1 & - \\
\hline 6 & Floraí & 1 & - \\
\hline 7 & Ivaiporã & 1 & - \\
\hline 8 & Jaguariaíva & 1 & - \\
\hline 9 & Pato Branco & 1 & - \\
\hline 10 & Pitanga & 1 & - \\
\hline 11 & Porecatu & 1 & - \\
\hline 12 & Prudentópolis & 1 & - \\
\hline 13 & $\begin{array}{c}\text { São Mateus do } \\
\text { Sul }\end{array}$ & 1 & - \\
\hline 14 & Wenceslau Braz & 1 & - \\
\hline 15 & Goioerê & 0,997 & Assaí; Pitanga; Prudentópolis; \\
\hline 16 & Capanema & 0,997 & $\begin{array}{l}\text { Ivaiporã; Assaí; Pitanga; } \\
\text { Prudentópolis; }\end{array}$ \\
\hline 17 & Irati & 0,993 & Ivaiporã; Assaí; Pitanga; \\
\hline 18 & Paranavaí & 0,989 & $\begin{array}{l}\text { Ivaiporã; Assaí; Pitanga; } \\
\text { Prudentópolis; }\end{array}$ \\
\hline 19 & Palmas & 0,989 & Ivaiporã; Assaí; Pitanga; \\
\hline 20 & Astorga & 0,988 & $\begin{array}{l}\text { Ivaiporã; Assaí; Pitanga; } \\
\text { Prudentópolis; }\end{array}$ \\
\hline 21 & Jacarezinho & 0,986 & Ivaiporã; Assaí; Pitanga; \\
\hline 22 & Cornélio Procópio & 0,985 & $\begin{array}{l}\text { Ivaiporã; Assaí; Pitanga; } \\
\text { Prudentópolis; }\end{array}$ \\
\hline 23 & Ibaiti & 0,985 & $\begin{array}{l}\text { Porecatu; Faxinal; Pitanga; } \\
\text { Prudentópolis; }\end{array}$ \\
\hline 24 & Rio Negro PR & 0,984 & Faxinal; Assaí; Prudentópolis; \\
\hline 25 & União Vitória & 0,980 & $\begin{array}{l}\text { Faxinal; Assaí; Pitanga; } \\
\text { Prudentópolis; }\end{array}$ \\
\hline 26 & Telêmaco Borba & 0,979 & Ivaiporã; Assaí; Pitanga; \\
\hline 27 & Paranaguá & 0,977 & Faxinal; Assaí; Prudentópolis; \\
\hline 28 & Umuarama & 0,973 & Ivaiporã; Assaí; \\
\hline 29 & Lapa & 0,920 & Faxinal; \\
\hline
\end{tabular}

Fonte: dados da pesquisa (2015).

partir da Tabela 5, constata-se que dentre as microrregiões analisadas no Grupo 3, cujos recursos físicos disponibilizados ao atendimento do SUS, têm sido utilizados de forma satisfatória por $48,27 \%$, ou seja, obtiveram scores de eficiência máxima. Também que os demais $51,73 \%$ estão próximos da fronteira de eficiência, contribuindo assim com a qualidade de vida da população do estado do Paraná.

Contudo, nota-se, tanto no Grupo 2 quanto no Grupo 3, a homogeneidade no que tange a eficiência das microrregiões de saúde analisadas, sendo que no Grupo 2, cinco apresentaram scores acima da média $(0,988)$ e no Grupo 3 , dezesseis acima da média $(0,990)$ e que os benchmarks principais foram as microrregiões de Ivaiporã, Assaí, Pitanga, Prudentópolis e Faxinal. Ressalta-se que em ambos os grupos todas as microrregiões analisadas estão próximos à fronteira de eficiência, corroborando com os resultados de Al-Shammari (1999) em que dentre os hospitais pesquisados, a maior parte, demonstraram-se eficientes e que os demais, sendo menos eficientes, estão muito próximos à fronteira de eficiência.

A Tabela 6 a seguir expressa os scores de eficiência e os benchmarks dos hospitais agrupando os 3 grupos de clusters.

Tabela 6: Eficiência de todas as microrregiões

\begin{tabular}{|c|c|c|c|}
\hline Ordem & Microrregião & $\begin{array}{c}\text { Score de } \\
\text { Eficiência }\end{array}$ & Benchmark \\
\hline 1 & Assaí & 1 & - \\
\hline 2 & Cerro Azul & 1 & - \\
\hline 3 & Faxinal & 1 & - \\
\hline 4 & Floraí & 1 & - \\
\hline 5 & Francisco Beltrão & 1 & - \\
\hline 6 & Ivaiporã & 1 & - \\
\hline 7 & Jaguariaíva & 1 & - \\
\hline 8 & Pato Branco & 1 & - \\
\hline 9 & Pitanga & 1 & - \\
\hline 10 & Porecatu & 1 & - \\
\hline 11 & Prudentópolis & 1 & - \\
\hline 12 & São Mateus do Sul & 1 & - \\
\hline 13 & Wenceslau Braz & 1 & - \\
\hline 14 & Cianorte & 0,998 & Ivaiporã; Francisco Beltrão; Pitanga; Prudentópolis; \\
\hline 15 & Goioerê & 0,997 & Francisco Beltrão; Pitanga; Prudentópolis; \\
\hline 16 & Capanema & 0,996 & Ivaiporã; Assaí; Francisco Beltrão; Pitanga; Prudentópolis; \\
\hline 17 & Toledo & 0,993 & Assaí; Francisco Beltrão; Prudentópolis; \\
\hline 18 & Campo Mourão & 0,991 & Assaí; Francisco Beltrão; Pitanga; \\
\hline 19 & Irati & 0,991 & Assaí; Francisco Beltrão; Pitanga; \\
\hline 20 & Guarapuava & 0,989 & Assaí; Francisco Beltrão; \\
\hline 21 & Palmas & 0,988 & Assaí; Francisco Beltrão; Pitanga; \\
\hline 22 & Paranavaí & 0,988 & Assaí; Prudentópolis; \\
\hline 23 & Astorga & 0,987 & Assaí; Francisco Beltrão; Prudentópolis; \\
\hline 24 & Apucarana & 0,986 & Assaí; Francisco Beltrão; \\
\hline 25 & Jacarezinho & 0,985 & Assaí; Francisco Beltrão; Pitanga; Prudentópolis; \\
\hline 26 & Cascavel PR & 0,985 & Assaí; Francisco Beltrão; \\
\hline 27 & Ibaiti & 0,985 & Porecatu; Faxinal; Pitanga; Prudentópolis; \\
\hline 28 & Foz do Iguaçu & 0,984 & Assaí; Francisco Beltrão; \\
\hline 29 & Rio Negro PR & 0,984 & Faxinal; Assaí; Pitanga; Prudentópolis; \\
\hline 30 & Cornélio Procópio & 0,984 & Assaí; Francisco Beltrão; Pitanga; \\
\hline 31 & União Vitória & 0,980 & Faxinal; Assaí; Prudentópolis; \\
\hline 32 & Telêmaco Borba & 0,979 & Assaí; Francisco Beltrão; Pitanga; \\
\hline 33 & Paranaguá & 0,977 & Faxinal; Assaí; Prudentópolis; \\
\hline 34 & Maringá & 0,976 & Assaí; Francisco Beltrão; \\
\hline 35 & Curitiba & 0,975 & Assaí; Francisco Beltrão; \\
\hline 36 & Umuarama & 0,971 & Assaí; Francisco Beltrão; \\
\hline 37 & Ponta Grossa & 0,968 & Assaí; Francisco Beltrão; \\
\hline 38 & Londrina & 0,960 & Assaí; Francisco Beltrão; \\
\hline 39 & Lapa & 0,920 & Faxinal; \\
\hline
\end{tabular}

Fonte: Dados da pesquisa (2015).

Verifica-se que, dentre todas as microrregiões analisadas no estudo, incluindo os 3 Grupos, os recursos físicos disponibilizados ao atendimento do SUS, têm sido utilizados de forma satisfatória por praticamente todas, visto que $33,33 \%$ apresentaram score de 
eficiência 1 , e as $66,67 \%$ restantes estão muito próximas da fronteira de eficiência, sendo que o menor score evidenciado foi de 0,920.
A Tabela 7 expõe o resumo dos resultados encontrados a partir da análise dos 3 Grupos.

Tabela 7: Resumo dos resultados

\begin{tabular}{l|c|c|c}
\hline \multicolumn{1}{c|}{ Cluster } & Microrregiões Analisadas & DMU's Eficientes & Menor Eficiência \\
\hline Grupo 1 & 1 & 1 & 1 \\
\hline Grupo 2 & 9 & 2 & 0,968 \\
\hline Grupo 3 & 29 & 14 & 0,920 \\
\hline Total & $\mathbf{3 9}$ & $\mathbf{1 7}$ & - \\
\hline
\end{tabular}

Fonte: Dados da pesquisa (2015).

Expresso na Tabela 7 conclui-se que $43,59 \%$ das microrregiões analisadas são eficientes e utilizam de forma eficiente os recursos físicos disponíveis ao atendimento da população pelo SUS. Neste contexto, para as DMU's que não atingiram o score máximo de eficiência, Kirigia et al. (2004) discorre opções para melhoria, como por exemplo, a redução do pessoal ligado à área administrativa, a transferência de equipamentos dos centros ineficientes para aqueles considerados eficientes, bem como sua venda ou locação para empresas privadas.

Os resultados podem estar atrelados à boa gestão dos recursos repassados e utilizados ao atendimento SUS, corroborando com achados de Kaveski et al. (2015), os quais indicam que 31\% dos municípios da amostra são eficientes e constataram a eficiência da utilização dos recursos destinados às unidades de saúde vinculadas ao SUS pertencentes ao Sudoeste do Mato Grosso do Sul é satisfatória.

No entanto, os resultados encontrados por Souza et al. (2010) são controversos aos deste estudo, pois inferiram que hospitais menos eficientes estão presentes nos municípios com maior número de habitantes, e nos municípios com número menor de habitantes estão os hospitais mais eficientes. Neste sentido, pode-se comparar a análise efetuada para o Grupo 1, em que se avaliou todos os municípios da microrregião de Curitiba, e ambos apresentaram eficiência máxima. Além disso, os resultados podem ser contrastados aos de Santos et al. (2016), os quais demonstraram um desempenho mediano das microrregiões do Sudeste, sendo possível inferir a existência de diferentes modelos de politicas públicas utilizados.

\section{CONSIDERAÇÕES FINAIS}

Movido pelo objetivo de avaliar, por meio da Análise Envoltória de Dados (DEA), a eficiência dos hospitais que atendem pelo Sistema Único de Saúde (SUS) das microrregiões do estado do Paraná, realizou-se uma pesquisa descritiva com abordagem quantitativa por meio de pesquisa documental. A população é composta por 416 hospitais de 39 microrregiões de saúde, contemplando todos os municípios do estado do Paraná. Dos 399 municípios, apenas 219 continham hospitais com disponibilidade de todas as variáveis necessárias para análise, contudo a amostra da pesquisa permaneceu com 39 microrregiões.

A fim de classificar as microrregiões de forma a não efetuar comparações indevidas, foram formados grupos levando em consideração a semelhança dos inputs de cada microrregião por meio da técnica de clusters. Formou-se, com isso, 3 grupos com 1 , 9 e 29 microrregiões, respectivamente.

Quanto ao Grupo 1, na microrregião de Curitiba, evidenciou-se a eficiência com score 1 para os treze municípios que a compõe. Dentre as nove microrregiões que compõe o Grupo 2, duas se apresentaram com score 1 de eficiência, 7 foram menos eficientes, no entanto estão muito próximas à fronteira de eficiência. Para este grupo, os principais benchmarks foram as microrregiões de Toledo e Francisco Beltrão. No que tange a eficiência das microrregiões do Grupo 3, 48,27\%, apresentaram scores de eficiência máxima e as demais $51,73 \%$ estão muito próximas da fronteira de eficiência, e os benchmarks principais foram as microrregiões de Ivaiporã, Assaí, Pitanga, Prudentópolis e Faxinal.

Vale ressaltar que em ambos os grupos analisados não foi identificada discrepância significativa quanto aos scores de eficiência nas microrregiões menos eficientes, ou seja, que não atingiram eficiência máxima. Conclui-se que praticamente metade das microrregiões analisadas são eficientes, sendo que, nas demais microrregiões, a maioria dos hospitais estão próximos à fronteira ou possuem um bom grau de eficiência, isso indica que a capacidade de otimização dos recursos está no limite, exigindo maiores investimentos, contribuindo, assim, com a qualidade de vida da população do estado do Paraná.

Conclui-se que os hospitais, de maneira geral, estão sendo geridos de forma eficiente no que tange os recursos repassados e utilizados ao atendimento SUS, podendo-se inferir que os repasses de recursos do SUS reduzem a ineficiência, reflexos dos avanços alcançados pela gestão do SUS. Por fim, futuras pesquisas podem ser efetuadas a partir da aplicação deste escopo de pesquisa, porém com intenção de evidenciar o envolvimento das políticas quanto à gestão dos recursos aplicados à saúde pública. Variáveis externas podem ser incluídas e também outras regiões e estados podem ser explorados a fim de proceder a comparações com o presente estudo.

\section{REFERÊNCIAS}

Al-Shammari, M. (1999). A multi-criteria data envelopment analysis model for measuring the productive efficiency of hospitals. International Journal of Operations \& Production Management, 19(9), 879-891.

Andrews, R., \& Entwistle, T. (2010). Does cross-sectoral partnership deliver? An empirical exploration of public service effectiveness, efficiency, and equity. Journal of Public Administration Research and Theory, 045.

Behn, R. D. (1998). O novo paradigma da gestão pública e a busca da accountability democrática. Revista do Serviço Público, 49(4).

Castelar, R. M., Mordelet, P., \& Grabois, V. (1995). Gestão hospitalar: um desafio para o hospital brasileiro. In Gestão hospitalar: um desafio para o hospital brasileiro. ENSP.

Cebrián, S. R. (1995). Glossário de economia de la salud. Madrid: Diaz de Santos.

Cesconetto, A., Lapa, J.S., \& Calvo, M. C. M. (2008). Avaliação da eficiência produtiva de hospitais do SUS de Santa Catarina, Brasil Evaluation of 
productive efficiency in the Unified National Health System hospitals in the State of Santa Catarina, Brazil. Cadernos de Saúde pública, 24(10), 2407 2417.

Chuang, C. L., Chang, P. C., \& Lin, R. H. (2011). An efficiency data envelopment analysis model reinforced by classification and regression tree for hospital performance evaluation. Journal of medical systems, 35(5), 10751083.

Clement, J. P., Valdmanis, V. G., Bazzoli, G. J., Zhao, M., \& Chukmaitov, A. (2008). Is more better? An analysis of hospital outcomes and efficiency with a DEA model of output congestion. Health Care Management Science, 11(1), 67-77.

CNESNet - Secretaria de Atenção à Saúde (2015). Tipos de Estabelecimentos. Recuperado em 20 de Novembro, 2015, de http://cnes2.datasus.gov.br/Mod_Ind_Unidade.asp.

Corrar, L. J., Paulo, E., \& Dias Filho, J. M. (2007). Análise multivariada: para os cursos de administração, ciências contábeis e economia. São Paulo: Atlas, 280-323.

Datasus - Portal da Saúde (2015). Epidemiológicas e Morbidade. Recuperado em 26 de novembro, 2015, de http://www2.datasus.gov.br/DATASUS/index.php?area=0203.

Datasus - Portal da Saúde (2015). Equipamentos. Recuperado em 26 de novembro, 2015 ,

http//tabnet.datasus.gov.br/cgi/deftohtm.exe?cnes/cnv/equipopr.def.

Datasus - Portal da Saúde (2015). Procedimentos Hospitalares do SUS. Recuperado em 26 de novembro, 2015, de http://www2.datasus.gov.br/DATASUS/index.php?area=0203.

Datasus - Portal da Saúde (2015). Procedimentos Hospitalares do SUS Recuperado em 27 de novembro, 2015, de http://tabnet.datasus.gov.br/cgi/deftohtm.exe?sih/cnv/qirs.def.

Fadel, M. A. V., \& Regis Filho, G. I. (2009). Percepção da qualidade em serviços públicos de saúde: um estudo de caso. Revista de Administração Pública, 43(1), 7-22.

Fonseca, P. C., \& Ferreira, M. A. M. (2009). Investigação dos níveis de eficiência na utilização de recursos no setor de saúde: uma análise das microrregiões de Minas Gerais. Saúde e Sociedade, 18(2), 199-213.

Greiling, D. (2006). Performance measurement: a remedy for increasing the efficiency of public services? International Journal of Productivity and Performance Management, 55(6), 448-465

Haas, D. J. (2003). Productive efficiency of English football teams-a data envelopment analysis approach. Managerial and Decision Economics, 24(5) 403-410.

Hames, D. S. (1991). Productivity-enhancing work innovations: Remedies for what ails hospitals? Journal of Healthcare Management, 36(4), 545.

Hu, H. H., Qi, Q., \& Yang, C. H. (2012). Analysis of hospital technical efficiency in China: Effect of health insurance reform. China Economic Review, 23(4) 865-877.

Jacobs, R. (2001). Alternative methods to examine hospital efficiency: data envelopment analysis and stochastic frontier analysis. Health Care Management Science, 4(2), 103-115.

Katz, D., \& Kahn, R. L. (1975). Psicologia social das organizações. São Paulo: Atlas, 2(1).

Kaveski, S., David, I., Degenhart, L., Vogt, M., \& Hein, N. (2015). A Eficiência do Atendimento dos municípios vinculados ao SUS no Sudoeste de Mato Grosso do Sul. Administração Pública e Gestão Social, 7(4).

Kirigia, J. M., Emrouznejad, A., Sambo, L. G., Munguti, N., \& Liambila, W (2004). Using data envelopment analysis to measure the technical efficiency of public health centers in Kenya. Journal of Medical Systems, 28(2), 155-166. Lins, M. P. E., Meza, L. A. (2000) Análise Envoltória de Dados e Perspectivas de Integração no Ambiente de Apoio à Decisão. Rio de Janeiro: COPPE/UFRJ. Macedo, M. A. S., Barbosa, A. C. T. A. M., \& Cavalcante, G. T. (2009) Desempenho de Agências Bancárias no Brasil: aplicando Análise Envoltória de Dados (DEA) a indicadores relacionados às perspectivas do BSC. Revista Economia \& Gestão, 9(19), 65-84.
Malik, A. M., \& Teles, J. P. (2001). Hospitais e programas de qualidade no Estado de São Paulo. Revista de Administração de Empresas, 41(3), 51-59.

Marinho, A. (2003). Avaliação da eficiência técnica nos serviços de saúde nos municípios do Estado do Rio de Janeiro. Revista brasileira de economia, 57(3) 515-534.

Marinho, A. (2001). Estudo de eficiência em alguns hospitais públicos e privados com a geração de rankings. Instituto de Pesquisa Econômica Aplicada, 794.

Mello, J. C. C. B. S., Meza, L. A., Gomes, E. G., \& Biondi Neto, L. (2005). Curso de análise de envoltória de dados. In: Simpósio Brasileiro de Pesquisa Operacional, Gramado, RS, Brasil, 37.

Mills, A. E., \& Spencer, E. M. (2005). Values based decision making: a tool for achieving the goals of healthcare. In HEC Forum (Vol. 17, No. 1, pp. 18-32) Kluwer Academic Publishers.

Moreira, S. (2008). Análise da eficiência dos hospitais-empresa: uma aplicação da data envelopment analysis. Boletim Económico-Banco de Portugal, 14(1), 127-150.

Morrisey, M. A., Wedig, G. J., \& Hassan, M. (1996). Do nonprofit hospitals pay their way?. Health Affairs, 15(4), 132-144.

Mwita, J. I. (2000). Performance management model: a systems-based approach to public service quality. International Journal of Public Sector Management, 13(1), 19-37.

Nunes, A. M., \& Harfouche, A. P. J. (2015). A reforma da administração pública aplicada ao setor da saúde: a experiência portuguesa. Revista de Gestão em Sistemas de Saúde, 4(2), 1-8.

Oro, I. M., Beuren, I. M., \& Hein, N. (2009). Análise da Eficiência de Empresas Familiares Brasileiras. RAE-eletrônica, 8(2)

Politelo, L., Rigo, V. P., \& Hein, N. (2015). Eficiência da Aplicação de Recursos no Atendimento do Sistema Único de Saúde (SUS) nas Cidades de Santa Catarina. Revista de Gestão em Sistemas de Saúde, 3(2), 45-60.

Politelo, L., \& Scarpin, J. E. (2013). Eficiência do atendimento do SUS nas microrregiões do estado de Santa Catarina. RAHIS, 10(1).

Richardson, R. J. (1999). Pesquisa social: métodos e técnicas. São Paulo. Editora Atlas. 3.

Sanderson, I. (1996). Evaluation, learning and the effectiveness of public services: Towards a quality of public service model. International Journal of Public Sector Management, 9(5/6), 90-108.

Santos, L. M., Souza Francisco, J. R., \& Gonçalves, M. A. (2016). Controle na alocação de recursos na saúde pública: uma análise nas microrregiões do sudeste brasileiro. Administração Pública e Gestão Social, 1(2), 119-130.

Shaw, C. D. (2003). Evaluating accreditation. International Journal for Quality in Health Care, 15(6), 455-456.

Simon, H. A. (1957). Administrative Behavior: A study of decision-making processes in adminstrative organization. New York: Free Press. 2(1), 259

Smith, P. C., \& Street, A. (2005). Measuring the efficiency of public services: the limits of analysis. Journal of the Royal Statistical Society: Series A (Statistics in Society), 168(2), 401-417.

Souza, I. V., Nishijima, M., \& Rocha, F. (2010). Eficiência do setor hospitalar nos municípios paulistas. Economia aplicada, 14(1), 51-66.

Souza, P. C. D., \& Scatena, J. H. G. (2010). Aplicação da gestão de custos para o aumento na eficiência dos hospitais públicos. Revista administração em saúde, 12(49), 195-207.

Vergara, S. C. (2000). Começando a definir a metodologia. Projetos e relatórios de pesquisa em administração, 3, 46-53.

Viacava, F., Ugá, M. A., Porto, S., Laguardia, J., \& Moreira, R. S. (2004). Avaliação de Desempenho de Sistemas de Saúde: um modelo de análise/Evaluation of performance of health systems: a model for analysis. Ciênc. saúde coletiva, 17(4), 921-934.

Wolff, L. D. G. (2005). Um modelo para avaliar o impacto do ambiente operacional na produtividade de hospitais brasileiros (Doctoral dissertation, Universidade Federal de Santa Catarina, Centro Tecnológico. Programa de Pós-Graduação em Engenharia de Produção.). Florianópolis, SC, Brasil. 